\title{
Synthesis and Antiproliferative activity in vitro of Amidino Substituted 2-phenylbenzazoles
}

\author{
Livio Racané, ${ }^{1}$ Kristina Butković, ${ }^{2}$ Irena Martin-Kleiner, ${ }^{3}$ Marijeta Kralj, ${ }^{3}$ Grace Karminski-Zamola, ${ }^{2}$ Marijana Hranjec ${ }^{2, *}$
}

\footnotetext{
1 Department of Applied Chemistry, Faculty of Textile Technology, University of Zagreb, Baruna Filipovića 28a, HR-10000 Zagreb, Croatia

2 Department of Organic Chemistry, Faculty of Chemical Engineering and Technology, University of Zagreb, Marulićev trg 20, HR-10000 Zagreb, Croatia

3 Division of Molecular Medicine, Ruđer Bošković Institute, Bijenička cesta 54, P. O. Box 180, HR-10000 Zagreb, Croatia

* Corresponding author's e-mail address: mhranjec@fkit.hr
}

RECEIVED: May 31, 2019 * REVISED: July 17, 2019 * ACCEPTED: July 18, 2019

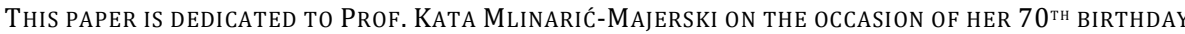

\begin{abstract}
Within this work we describe the synthesis of versatile substituted 2-phenyl benzothiazole 3-10 and 2-phenylbenzimidazole 12-19 derivatives bearing amidino groups. Furthermore, the synthesized compounds were explored for their antiproliferative activity in vitro on three cancer cell lines. Tested compounds showed moderate to strong antiproliferative activity. Furthermore, the type of the attached amidino group on benzazole nuclei has the significant impact on the antiproliferative activity only within benzimidazole derivatives with 2-imidazolinyl substituted derivatives being more active in comparison to amidino substituted analogues. All obtained results revealed that this type of benzothiazole derivatives have a great potential for further optimization and development of more efficient potential antiproliferative agents.
\end{abstract}

Keywords: amidines, benzimidazoles, benzothiazoles, antiproliferative activity in vitro.

\section{INTRODUCTION}

D UE to their biological importance based on a broad spectrum of biological features, benzimidazole and benzothiazole derivatives are nowadays important and well-known privileged building structural motifs in medicinal and pharmaceutical chemistry. ${ }^{[1,2]}$ These nitrogen and sulphur-containing heterocycles become unavoidable substructures in the rational design of novel drugs. ${ }^{[3,4]}$ These derivatives has been proven to possess important role in the structure of various biologically important natural and synthetic molecules, while among their versatile pharmacological features, the most important ones are antimicrobial, ${ }^{[5,6]}$ antitumor, ${ }^{[7,8]}$ antiviral, ${ }^{[9]}$ anti-inflammanory, ${ }^{[10]}$ antihistaminic, ${ }^{[11]}$ antioxidant, ${ }^{[12,13]}$ etc. Importantly, since benzimidazoles are bioisosteres of purines, which offer the possibility of interactions with biomolecules from the living systems, benzimidazole derivatives play a crucial role in the function of many biologically important molecules like DNA, RNA or different proteins included in numerous metabolic processes in living organisms. ${ }^{[14,15]}$
Recently, we have published several papers which describe the antiproliferative activity and potential of versatile benzothiazole and benzimidazole derivatives substituted with carboxamido, amino, halogeno, cyano, amidino, amino or nitro groups placed at different positions on the mentioned scaffold. The most significant biological importance was observed with amidino substituted benzazoles bearing different types of amidine substituents suchlike unsubstituted, isopropyl, morpholinyl or imidazolinyl. ${ }^{[16]}$ Obtained results revealed that the most significant influence and the enhancement of the antiproliferative activity in vitro showed benzazole derivatives substituted with cyclic amidino substituent, namely 2-imidazolinyl group with $\mathrm{IC}_{50}$ values in submicromolar range of concentrations (Fig. 1). ${ }^{[17,18]}$ Interestingly, some of the tested derivatives showed selectivity towards cancer cells as well as very low cytotoxicity against normal fibroblasts.

Additionally, we have also explored the interaction with DNA/RNA of chosen amidino substituted benzothiazoles/benzimidazoles in order to define one of the possible mechanisms of biological action of most active derivatives. The results clearly indicated that amidino 

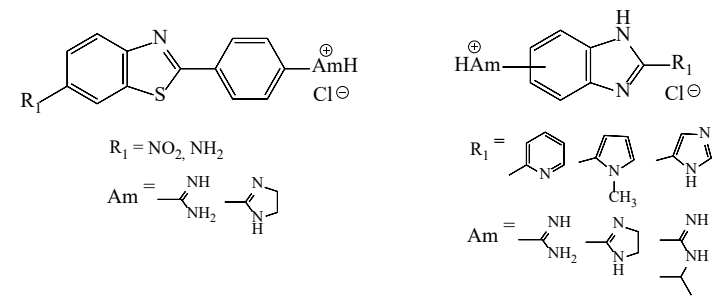

Figure 1. Previously published amidino substituted benzothiazole and benzimidazole derivatives with antiproliferative activity.

substituents which are usually placed at the termini of the molecules have great importance in the interaction with biological targets causing enhanced antitumor activity and stronger interaction with biomacromolecules. ${ }^{[19-21]}$

Amidines seem to contribute significantly to the molecule/possible biological target complex stability through $\mathrm{H}$-bonding and electrostatic interactions. ${ }^{[22,23]}$ Thus, in several publications we have proved that by incorporation of positively charged amidine moiety at the end of the heteroaromatic molecules, we could significantly improve the biological activity and orient the function of the molecule toward the binding to an electronegatively charged molecule such as DNA/RNA. ${ }^{[24]}$ Studied benzothiazole derivatives bearing amidine moiety intercalate into ds-RNA by binding into minor groove of ATDNA, and agglomerate along GC-DNA (Fig. 2). Furthermore, benzothiazoles also interact with ss-RNA, but only 2imidazolinyl 2-phenylbenzothiazole displayed well defined orientation and dominant binding mode (by induced $C D$ signals) with poly A and poly G. ${ }^{[25]}$ Amidino substituted benzimidazole-2-carboxamide as groove binder evidenced sequence-selective binding in the A-T rich side (Fig. 2). ${ }^{[26]}$

As a continuation of our scientific research based on the synthesis of biologically active benzazoles, all the above-mentioned facts prompted us to design and synthesize novel amidino substituted benzothiazole / enzimidazole derivatives in order to evaluate their antiproliferative activity in vitro. The obtained results are discussed in terms of SAR to define the influence of the type of amidino group and heteroatom placed in the heterocyclic nuclei as well as the type of substituent on the phenyl ring on the antiproliferative activity.
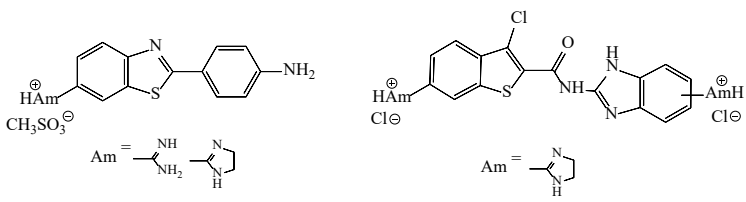

Figure 2. Previously published amidino substituted benzothiazole and benzimidazole derivatives with antiproliferative activity and interaction with DNA/RNA.

\section{EXPERIMENTAL}

\section{Chemistry \\ GENERAL METHODS}

All chemicals and solvents were purchased from commercial suppliers including Aldrich, Fluka and Acros. Melting points were recorded on an Original Kofler Mikroheitztisch apparatus (Reichert, Wien) and SMP11 Bibby apparatus and are not corrected. All NMR spectra were measured in DMSO- $d_{6}$ solutions using TMS as an internal standard. The ${ }^{1} \mathrm{H}$ NMR and the ${ }^{13} \mathrm{C}$ NMR spectra were recorded with the Bruker Avance DPX-300, Bruker AV600 or Bruker Avance III HD 400. Chemical shifts are reported in parts per million (ppm) relative to TMS. LC-MS was performed on the Waters Acquity UHPLC system.

\section{Synthesis}

Synthesis of main precursors for the synthesis of targeted benzothiazoles and benzimidazoles, namely 2-amino-5amidiniumbenzenethiolate $\quad \mathbf{2}^{[27]}$ and 2-amino-(4,5dihydro- $1 \mathrm{H}$-imidazol-3-ium-2-yl)benzenethiolate hydrate $\mathbf{2 b}^{[27]}$ and 4-amidinium-1,2-phenylenediamine chloride 11a and 2-(3,4-diaminophenyl)-4,5-dihydro- $1 \mathrm{H}$-imidazol-3-ium chloride 11b was carried out according to the previously published experimental procedures. ${ }^{[28]}$

\section{GENERAL METHOD FOR THE SYNTHESIS OF BENZOTHIAZOLE DERIVATIVES 3-10}

A mixture of the corresponding aldehyde $1 \mathbf{a}-\mathbf{1 d}(0.5 \mathrm{mmol})$ and 2-amino-5-amidiniumbenzenethiolate $2 \mathrm{a}(0.5 \mathrm{mmol})$ or 2-amino-5-(4,5-dihydro-1H-imidazol-3-ium-2-yl)benzenethiolate hydrate $\mathbf{2 b}(0.5 \mathrm{mmol})$ in glacial acetic acid ( 5 $\mathrm{mL}$ ) was stirred at reflux under nitrogen for $3 \mathrm{~h}$, followed by the addition of concentrated hydrochloric acid $(0.5 \mathrm{~mL})$ and additionally stirred at room temperature for $1 \mathrm{~h}$. After addition of diethyl ether, the crude product was filtered off, washed with diethyl ether and crystallized from $0.1 \mathrm{M}$ hydrochloric acid/acetone mixture to obtain pure compounds 3-10.

6-Amidinium-2-(4-cyanophenyl)benzothiazole Chloride 3 Using above described method from 4-cyanobenzaldehyde 1a $(0.066 \mathrm{~g}, 0.5 \mathrm{mmol})$ and 2-amino-5-amidiniumben-zenethiolate $2 \mathrm{a}(0.084 \mathrm{~g}, 0.5 \mathrm{mmol})$ was obtained $0.088 \mathrm{~g}(52 \%)$ of pale yellow solid; $\mathrm{mp}=291-$ $295{ }^{\circ} \mathrm{C} ;{ }^{1} \mathrm{H}$ NMR (400 MHz, DMSO- $\left.d_{6}\right) \delta / \mathrm{ppm}$ : 9.64 (bs, $\left.2 \mathrm{H},-\mathrm{C}\left(\mathrm{NH}_{2}\right)_{2}{ }^{+}\right), 9.45\left(\mathrm{bs}, 2 \mathrm{H},-\mathrm{C}\left(\mathrm{NH}_{2}\right)_{2}{ }^{+}\right), 8.79(\mathrm{~s}, 1 \mathrm{H}, \mathrm{Ar}-\mathrm{H})$, $8.33(\mathrm{~m}, 3 \mathrm{H}, \mathrm{Ar}-\mathrm{H}), 8.08$ (d, $2 \mathrm{H}, J=7.2 \mathrm{~Hz}, \mathrm{Ar}-H), 8.00$ (d, $1 \mathrm{H}, J=7.7 \mathrm{~Hz}, \operatorname{Ar}-H) ;{ }^{13} \mathrm{C}$ NMR $\left(100 \mathrm{MHz}, \mathrm{DMSO}-d_{6}\right)$ ס/ppm: 169.5, 165.4, 156.2, 136.0, 135.1, 133.4 (2C), 128.2 (2C), 126.6, 125.5, 123.7, 123.5, 118.2, 114.1; LCMS (ESI) $m / z: 279.2\left[\left(\mathrm{M}-\mathrm{Cl}^{-}\right)^{+}\right]$. 
6-Amidinium-2-(4-chlorophenyl)benzothiazole chloride 4 Using above described method from 4-chlorobenzaldehyde 1b $(0.075 \mathrm{~g}, 0.5 \mathrm{mmol})$ and 2-amino-5-amidiniumbenzenethiolate $2 \mathrm{a}(0.084 \mathrm{~g}, 0.5 \mathrm{mmol})$ was obtained $0.091 \mathrm{~g}$ (56\%) of colorless solid; $\mathrm{mp}=299-302{ }^{\circ} \mathrm{C} ;{ }^{1} \mathrm{H}$ NMR $(600 \mathrm{MHz}$, DMSO- $\left.d_{6}\right) \delta / \mathrm{ppm}: 9.41\left(\mathrm{bs}, 4 \mathrm{H},-\mathrm{C}\left(\mathrm{NH}_{2}\right)_{2}{ }^{+}\right), 8.72(\mathrm{~s}, 1 \mathrm{H}, \mathrm{Ar}-\mathrm{H})$, $8.27(\mathrm{~d}, 1 \mathrm{H}, J=7.2 \mathrm{~Hz}, \mathrm{Ar}-\mathrm{H}), 8.18(\mathrm{~d}, 2 \mathrm{H}, J=6.5 \mathrm{~Hz}, \mathrm{Ar}-\mathrm{H})$, $7.95(\mathrm{~d}, 1 \mathrm{H}, J=7.1 \mathrm{~Hz}, \operatorname{Ar}-H), 7.69(\mathrm{~d}, 2 \mathrm{H}, J=6.8 \mathrm{~Hz}, \mathrm{Ar}-\mathrm{H}) ;{ }^{13} \mathrm{C}$ NMR $\left(100 \mathrm{MHz}, \mathrm{DMSO}-d_{6}\right) \delta /$ ppm: 170.2, 165.4, 156.4, 138.9, 134.8, 131.1, 129.6 (2C), 129.3 (2C), 126.5, 125.1, 123.5, 123.1; LC-MS (ESI) $m$ / z: $288.1\left[\left(\mathrm{M}-\mathrm{Cl}-^{-}\right)^{+}\right]$.

\section{6-Amidinium-2-(4-trifluoromethylphenyl)benzothiazole chloride 5}

Using above described method from 4-trifluormethylbenzaldehide 1c $(0.087 \mathrm{~g}, 0.5 \mathrm{mmol})$ and 2-amino-5amidiniumbenzenethiolate $2 \mathrm{a}(0.084 \mathrm{~g}, 0.5 \mathrm{mmol})$ was obtained $0.074 \mathrm{~g}(41 \%)$ of colorless solid; $\mathrm{mp}=287-291^{\circ} \mathrm{C}$; ${ }^{1} \mathrm{H}$ NMR (400 MHz, DMSO-d $\left.d_{6}\right) \delta / \mathrm{ppm}: 9.52$ (bs, 4H, $\left.\mathrm{C}\left(\mathrm{NH}_{2}\right)_{2}{ }^{+}\right), 8.78(\mathrm{~s}, 1 \mathrm{H}, \mathrm{Ar}-\mathrm{H}), 8.38(\mathrm{~d}, 2 \mathrm{H}, J=7.3 \mathrm{~Hz}, \mathrm{Ar}-\mathrm{H})$, $8.33(\mathrm{~d}, 1 \mathrm{H}, J=8.3 \mathrm{~Hz}, \mathrm{Ar}-H), 7.99(\mathrm{~m}, 3 \mathrm{H}, \mathrm{Ar}-H) ;{ }^{13} \mathrm{C} \mathrm{NMR}$ $\left(100 \mathrm{MHz}, \mathrm{DMSO}-d_{6}\right) \delta / \mathrm{ppm}: 169.7,165.4,156.3,135.8$, $134.9,131.6\left({ }^{2} J_{\mathrm{CF}}=31.9 \mathrm{~Hz}\right), 128.4(2 \mathrm{C}), 126.6,126.5(2 \mathrm{C}$, $\left.{ }^{3} J_{\mathrm{CF}}=3.2 \mathrm{~Hz}\right), 125.5,123.8\left(2 \mathrm{C},{ }^{1} J_{\mathrm{CF}}=270.9 \mathrm{~Hz}\right), 123.7,123.4$; LC-MS (ESI) $m / z: 322.2\left[\left(\mathrm{M}-\mathrm{Cl}^{-}\right)^{+}\right]$.

\section{6-Amidinium-2-(2,4-dihydroxyphenyl)benzothiazole chloride $6^{[13]}$}

Using above described method from 2,4-dihydroxybenzaldehyde 1d (0.069 g, $0.5 \mathrm{mmol})$ and 2-amino-5amidiniumbenzenethiolate $2 \mathrm{a}(0.084 \mathrm{~g}, 0.5 \mathrm{mmol})$ was obtained $0.083 \mathrm{~g} \mathrm{(52 \% )}$ of pale yellow solid; $\mathrm{mp}>300{ }^{\circ} \mathrm{C}$. ${ }^{1} \mathrm{H}$ NMR (300 MHz, DMSO- $\left.d_{6}\right) \delta /$ ppm: $11.57(\mathrm{~s}, 1 \mathrm{H},-\mathrm{OH})$, $10.34(\mathrm{~s}, 1 \mathrm{H},-\mathrm{OH}), 9.42$ (bs, $\left.2 \mathrm{H},-\mathrm{C}\left(\mathrm{NH}_{2}\right)_{2}{ }^{+}\right), 9.14$ (bs, $2 \mathrm{H},-$ $\left.\mathrm{C}\left(\mathrm{NH}_{2}\right)_{2}{ }^{+}\right), 8.59(\mathrm{~s}, 1 \mathrm{H}, \mathrm{Ar}-\mathrm{H}), 8.14-8.10(\mathrm{~m}, 2 \mathrm{H}, \mathrm{Ar}-\mathrm{H}), 7.88$ $(\mathrm{d}, 1 \mathrm{H}, J=8.6 \mathrm{~Hz}, \operatorname{Ar}-H), 6.57(\mathrm{~s}, 1 \mathrm{H}, \mathrm{Ar}-H), 6.49$ (d, $1 \mathrm{H}, J=$ $8.7 \mathrm{~Hz}, \mathrm{Ar}-H)$; ${ }^{13} \mathrm{C}$ NMR $\left(75 \mathrm{MHz}, \mathrm{DMSO}-d_{6}\right) \delta / \mathrm{ppm}: 168.9$, $165.5,162.3,158.5,154.9,134.3,130.1,125.7,123.1$, 122.5, 121.2, 110.4, 108.6, 102.6; LC- MS (ESI) m / z: 286.1 $\left[\left(\mathrm{M}-\mathrm{Cl}^{-}\right)^{+}\right]$.

\section{6-(4,5-Dihydro-1H-imidazol-3-ium-2-yl)-2-(4- cyanophenyl)benzothiazole chloride 7}

Using above described method from 4-cyanobenzaldehyde 1a $(0.066 \mathrm{~g}, 0.5 \mathrm{mmol}$ ) and 2-amino-5-(4,5-dihydro- $1 \mathrm{H}$ imidazol-3-ium-2-yl)benzenethiolate hydrate $\mathbf{2 b}(0.106 \mathrm{~g}$, $0.5 \mathrm{mmol}$ ) was obtained $0.071 \mathrm{~g}$ (42\%) of pale yellow solid; $\mathrm{mp}>300{ }^{\circ} \mathrm{C} .{ }^{1} \mathrm{H}$ NMR $\left(600 \mathrm{MHz}, \mathrm{DMSO}-d_{6}\right) \delta / \mathrm{ppm}: 10.89$ $\left(\mathrm{s}, 2 \mathrm{H},-\mathrm{C}(\mathrm{NH}-)_{2}{ }^{+}\right), 8.94(\mathrm{~s}, 1 \mathrm{H}, \mathrm{Ar}-\mathrm{H}), 8.37(\mathrm{~d}, 1 \mathrm{H}, J=8.6 \mathrm{~Hz}$, Ar- $H$ ), $8.34(\mathrm{~d}, 2 \mathrm{H}, J=8.3 \mathrm{~Hz}, \mathrm{Ar}-\mathrm{H}), 8.16(\mathrm{~d}, 1 \mathrm{H}, J=8.6 \mathrm{~Hz}$, $\operatorname{Ar}-H), 8.09(\mathrm{~d}, 2 \mathrm{H}, J=8.3 \mathrm{~Hz}, \mathrm{Ar}-\mathrm{H}), 4.06\left(\mathrm{~s}, 4 \mathrm{H},-\mathrm{CH}_{2} \mathrm{CH}_{2}-\right.$ ); ${ }^{13} \mathrm{C}$ NMR (75 MHz, DMSO- $d_{6}$ ) $\delta /$ ppm: 170.1, 164.6, 156.6, 135.9, 135.4, 133.5 (2C), 128.3 (2C), 126.8, 124.1, 123.9,
119.6, 118.2, 114.2, 44.5 (2C); LC- MS (ESI) $m$ / z: 305.5 $\left[\left(\mathrm{M}-\mathrm{Cl}^{-}\right)^{+}\right]$.

\section{6-(4,5-Dihydro-1H-imidazol-3-ium-2-yl)-2-(4- chlorophenyl)benzothiazole chloride 8}

Using above described method from 4-chlorobenzaldehyde 1b $(0.075 \mathrm{~g}, 0.5 \mathrm{mmol})$ and 2-amino-5-(4,5-dihydro- $1 \mathrm{H}$ imidazol-3-ium-2-yl)benzenethiolate hydrate $\mathbf{2 b}(0.106 \mathrm{~g}$, $0.5 \mathrm{mmol}$ ) was obtained $0.071 \mathrm{~g}(42 \%)$ of colorless solid; $\mathrm{mp}>300{ }^{\circ} \mathrm{C} .{ }^{1} \mathrm{H}$ NMR $\left(600 \mathrm{MHz}\right.$, DMSO- $\left.d_{6}\right) \delta / \mathrm{ppm}: 10.81$ $\left(\mathrm{s}, 2 \mathrm{H},-\mathrm{C}(\mathrm{NH}-)_{2}{ }^{+}\right), 8.88(\mathrm{~s}, 1 \mathrm{H}, \mathrm{Ar}-\mathrm{H}), 8.32(\mathrm{~d}, 1 \mathrm{H}, J=8.6 \mathrm{~Hz}$, Ar-H), $8.19(\mathrm{~d}, 2 \mathrm{H}, J=8.3 \mathrm{~Hz}, \mathrm{Ar}-\mathrm{H}), 8.12(\mathrm{~d}, 1 \mathrm{H}, J=8.4 \mathrm{~Hz}$, Ar- $H$ ), $7.70(\mathrm{~d}, 2 \mathrm{H}, J=8.3 \mathrm{~Hz}, \mathrm{Ar}-\mathrm{H}), 4.06\left(\mathrm{~s}, 4 \mathrm{H},-\mathrm{CH}_{2} \mathrm{CH}_{2}-\right)$; ${ }^{13} \mathrm{C}$ NMR $\left(75 \mathrm{MHz}\right.$, DMSO- $\left.d_{6}\right) \delta / \mathrm{ppm:} \mathrm{170.8,} \mathrm{164.7,} \mathrm{156.8,}$ 137.1, 135.1, 131.0, 129.7 (2C), 129.4 (2C), 126.7, 123.8,

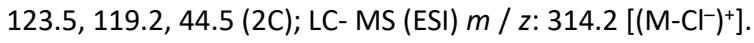

6-(4,5-Dihydro-1H-imidazol-3-ium-2-yl)-2-(4trifluoromethylphenyl)benzothiazole chloride 9

Using above described method from 4-trifluormethylbenzaldehide $1 \mathrm{c}(0.087 \mathrm{~g}, 0.5 \mathrm{mmol})$ and 2-amino-5-(4,5dihydro- $1 \mathrm{H}$-imidazol-3-ium-2-yl)benzenethiolate hydrate 2b (0.106 g, $0.5 \mathrm{mmol})$ was obtained $0.098 \mathrm{~g}$ (51 \%) of colorless solid; $\mathrm{mp}=264-267^{\circ} \mathrm{C}$. ${ }^{1} \mathrm{H}$ NMR $(600 \mathrm{MHz}$, DMSO$\left.d_{6}\right) \delta / \mathrm{ppm}: 10.86\left(\mathrm{~s}, 2 \mathrm{H},-\mathrm{C}(\mathrm{NH}-)_{2}{ }^{+}\right), 8.93(\mathrm{~s}, 1 \mathrm{H}, \mathrm{Ar}-\mathrm{H}), 8.38$ $(\mathrm{m}, 3 \mathrm{H}, \mathrm{Ar}-\mathrm{H}), 8.15(\mathrm{~d}, 1 \mathrm{H}, J=7.9 \mathrm{~Hz}, \mathrm{Ar}-\mathrm{H}), 7.99(\mathrm{~d}, 2 \mathrm{H}, J=$ $7.4 \mathrm{~Hz}, \mathrm{Ar}-\mathrm{H}), 4.06\left(\mathrm{~s}, 4 \mathrm{H},-\mathrm{CH}_{2} \mathrm{CH}_{2}-\right)$; ${ }^{13} \mathrm{C}$ NMR $(100 \mathrm{MHz}$, DMSO- $\left.d_{6}\right) \delta / \mathrm{ppm}: 170.2,164.3,156.5,135.6,135.1,131.7$ $\left({ }^{2} J_{\mathrm{CF}}=32.0 \mathrm{~Hz}\right), 128.4(2 \mathrm{C}), 126.9,126.4\left(2 \mathrm{C},{ }^{3} J_{\mathrm{CF}}=3.0 \mathrm{~Hz}\right)$, $124.1,123.8\left(2 \mathrm{C},{ }^{1} J_{\mathrm{CF}}=270.8 \mathrm{~Hz}\right), 123.7,119.4,44.5(2 \mathrm{C})$; LC-MS (ESI) $m / z: 348.2\left[\left(\mathrm{M}-\mathrm{Cl}^{-}\right)^{+}\right]$.

6-(4,5-Dihydro-1H-imidazol-3-ium-2-yl)-2-(2,4dihydroxyphenyl)benzothiazole chloride $10^{[13]}$ Using above described method from 2,4-dihydroxybenzaldehyde 1d (0.069 g, $0.5 \mathrm{mmol})$ and 2-amino-5-(4,5dihydro- $1 \mathrm{H}$-imidazol-3-ium-2-yl)benzenethiolate hydrate 2b (0.106 g, $0.5 \mathrm{mmol})$ was obtained $0.101 \mathrm{~g}(58 \%)$ of pale yellow solid; $\mathrm{mp}>300{ }^{\circ} \mathrm{C}$. ${ }^{1} \mathrm{H} \mathrm{NMR}\left(600 \mathrm{MHz}, \mathrm{DMSO}-d_{6}\right) \delta /$ ppm: $11.58(\mathrm{~s}, 1 \mathrm{H},-\mathrm{OH}), 10.69\left(\mathrm{~s}, 2 \mathrm{H},-\mathrm{C}(\mathrm{NH}-)_{2}{ }^{+}\right), 10.36(\mathrm{~s}$, $1 \mathrm{H},-\mathrm{OH}), 8.76(\mathrm{~d}, 1 \mathrm{H}, J=1.7 \mathrm{~Hz}, \mathrm{Ar}-\mathrm{H}), 8.16-8.13(\mathrm{~m}, 2 \mathrm{H}, \mathrm{Ar}-$ $H$ ), $8.04(\mathrm{dd}, 1 \mathrm{H}, J=8.6 \mathrm{~Hz}, J=1.9 \mathrm{~Hz}, \mathrm{Ar}-H), 6.58(\mathrm{~d}, 1 \mathrm{H}, J=$ $2.3 \mathrm{~Hz}, \mathrm{Ar}-H$ ), 6.49 (dd, $1 \mathrm{H}, J=8.8 \mathrm{~Hz}, J=2.3 \mathrm{~Hz}, \mathrm{Ar}-\mathrm{H}), 4.04$ (s, 4H, $\left.-\mathrm{CH}_{2} \mathrm{CH}_{2}-\right) ;{ }^{13} \mathrm{C}$ NMR (151 MHz, DMSO-d $\left.d_{6}\right) \delta / \mathrm{ppm}$ : $169.0,164.6,162.4,158.6,155.3,134.7,130.2,126.1$, 123.1, 121.6, 117.1, 110.6, 108.7, 102.6, 44.3 (2C); LC-MS (ESI) $\mathrm{m} / \mathrm{z}: 312.1\left[(\mathrm{M}-\mathrm{Cl})^{+}\right]$.

\section{GENERAL METHOD FOR THE SYNTHESIS OF BENZIMIDAZOLE DERIVATIVES 12-19}

A mixture of equimolar amounts of the corresponding aldehyde 1a-1d and 4-amidinium-1,2-phenylenediamine 11a or 2-(3,4-diaminophenyl)-4,5-dihydro-1H-imidazol-3-ium 
chloride $\mathbf{1 1 b}$ in absolute ethanol with $p$-benzoquinone was stirred at reflux for 4 hours. The crude product was then filtered off, washed with diethyl ether and recrystallized from ethanol to obtain pure compounds 12-19. [17]

\section{5(6)-Amidinium-2-(4-cyanophenyl) benzimidazole chloride 12}

Using above described method from 4-cyanobenzaldehyde 1a $(0.075 \mathrm{~g}, 0.6 \mathrm{mmol}), 4$-amidinium-1,2-phenylenediamine 11a $(0.107 \mathrm{~g}, 0.6 \mathrm{mmol})$ and $p$-benzoquinone $(0.062 \mathrm{~g}, 0.6 \mathrm{mmol})$ in absolute ethanol $(5 \mathrm{~mL})$ was obtained $0.148 \mathrm{~g}(87 \%)$ of grey solid; $\mathrm{mp}>300{ }^{\circ} \mathrm{C} ;{ }^{1} \mathrm{H}$ NMR $(300 \mathrm{MHz}$,

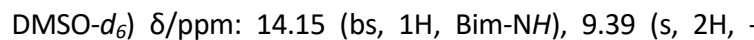
$\left.\mathrm{C}\left(\mathrm{NH}_{2}\right)_{2}{ }^{+}\right), 9.11\left(\mathrm{~s}, 2 \mathrm{H},-\mathrm{C}\left(\mathrm{NH}_{2}\right)_{2}{ }^{+}\right), 8.46(\mathrm{~d}, 2 \mathrm{H}, J=8.4 \mathrm{~Hz}, \mathrm{Ar}-\mathrm{H})$, $8.07(\mathrm{~d}, 2 \mathrm{H}, \mathrm{J}=8.8 \mathrm{~Hz}, \mathrm{Ar}-\mathrm{H}), 8.30(\mathrm{~s}, 1 \mathrm{H}, \mathrm{Ar}-\mathrm{H}), 7.83-7.67(\mathrm{~m}$, $2 \mathrm{H}, \mathrm{Ar}-H) ;{ }^{13} \mathrm{C}$ NMR $\left(75 \mathrm{MHz}\right.$, DMSO- $\left.d_{6}\right) \delta / \mathrm{ppm}: 166.7,166.4$, $150.5,133.9,133.6$ (2C), 129.8, 128.1 (2C), 123.4, 121.7, 120.5, 119.0, 113.3; LC-MS (ESI) m / z: 262.5 [(M-Cl-) $)^{+}$.

\section{5(6)-Amidinium-2-(4-chlorophenyl) benzimidazole chloride 13}

Using above described method from 4-chlorobenzaldehyde 1b $(0.075 \mathrm{~g}, 0.5 \mathrm{mmol})$, 4-amidinium-1,2-phenylenediamine 11a $(0.099 \mathrm{~g}, 0.5 \mathrm{mmol})$ and $p$-benzoquinone $(0.057 \mathrm{~g}$, $0.5 \mathrm{mmol})$ in absolute ethanol $(5 \mathrm{~mL})$ was obtained $0.118 \mathrm{~g}$ (72\%) of violet solid; mp $256-258{ }^{\circ} \mathrm{C}$; ${ }^{1} \mathrm{H}$ NMR $(300 \mathrm{MHz}$, DMSO- $d_{6}$ ) $\delta / \mathrm{ppm}: 13.84$ (bs, $1 \mathrm{H}, \mathrm{Bim}-\mathrm{NH}$ ), 9.37 (bs, $2 \mathrm{H}$, $\left.\mathrm{C}\left(\mathrm{NH}_{2}\right)_{2}{ }^{+}\right), 9.07\left(\mathrm{bs}, 2 \mathrm{H},-\mathrm{C}\left(\mathrm{NH}_{2}\right)_{2}{ }^{+}\right), 8.30(\mathrm{~d}, 2 \mathrm{H}, J=8.8 \mathrm{~Hz}, \mathrm{Ar}-$ $H), 8.19(\mathrm{~s}, 1 \mathrm{H}, \mathrm{Ar}-H), 7.80(\mathrm{~d}, 1 \mathrm{H}, J=7.6 \mathrm{~Hz}, \mathrm{Ar}-H), 7.70(\mathrm{~d}$, $1 \mathrm{H}, J=8.0 \mathrm{~Hz}, \mathrm{Ar}-H), 7.68(\mathrm{~d}, 2 \mathrm{H}, J=8.8 \mathrm{~Hz}, \mathrm{Ar}-H) ;{ }^{13} \mathrm{C} \mathrm{NMR}$ (75 MHz, DMSO- $d_{6}$ ) $\delta$ / ppm: 166.5, 166.4, 165.8, 153.7, 135.7, 129.7 (2C), 129.6, 129.2 (2C), 128.5, 122.7, 122.5, 121.9; LC-MS (ESI) m / z: $271.5\left[\left(\mathrm{M}-\mathrm{Cl}^{-}\right)^{+}\right]$.

\section{5(6)-Amidinium-2-(4-trifluoromethylphenyl)- benzimidazole chloride 14}

Using above described method from 4-trifluormethylbenzaldehide 1c $(0.075 \mathrm{~g}, 0.4 \mathrm{mmol}), 4$-amidinium-1,2phenylenediamine 11a $(0.080 \mathrm{~g}, 0.4 \mathrm{mmol})$ and $p$-benzoquinone $(0.046 \mathrm{~g}, 0.4 \mathrm{mmol})$ in absolute ethanol $(5 \mathrm{~mL})$ was obtained $0.098 \mathrm{~g}$ (67 \%) of dark grey solid; $\mathrm{mp} 252-254{ }^{\circ} \mathrm{C}$; ${ }^{1} \mathrm{H}$ NMR (600 MHz, DMSO-d $\left.d_{6}\right) \delta / \mathrm{ppm}: 14.02$ (s, 1H, Bim$\mathrm{NH}), 9.40\left(\mathrm{~s}, 2 \mathrm{H},-\mathrm{C}\left(\mathrm{NH}_{2}\right)_{2}{ }^{+}\right), 9.13\left(\mathrm{~s}, 2 \mathrm{H},-\mathrm{C}\left(\mathrm{NH}_{2}\right)_{2}{ }^{+}\right), 8.51(\mathrm{~d}$, $2 \mathrm{H}, J=8.4 \mathrm{~Hz}, \operatorname{Ar}-H), 8.23(\mathrm{~s}, 1 \mathrm{H}, \operatorname{Ar}-H), 7.98(\mathrm{~d}, 2 \mathrm{H}, J=8.4$ $\mathrm{Hz}, \mathrm{Ar}-\mathrm{H}), 7.83$ (bs, $1 \mathrm{H}, \mathrm{Ar}-\mathrm{H}), 7.73(\mathrm{~d}, 1 \mathrm{H}, J=8.0 \mathrm{~Hz}, \mathrm{Ar}-\mathrm{H}) ;{ }^{13} \mathrm{C}$ NMR (75 MHz, DMSO- $\left.d_{6}\right) \delta /$ ppm: 166.5, 165.8, 153.0, 133.6 130.7 (q), 128.2 (2C), 126.5 (2C), 125.9, 123.1, , 122.4, 120.5, 114.7, 113.3; LC-MS (ESI) m / z: 305.5 [(M-Cl-)'].

\section{5(6)-Amidinium-2-(2,4-dihydroxyphenyl)- benzimidazole chloride $15^{[13]}$}

Using above described method from 2,4-dihydroxybenz- aldehyde $1 \mathrm{~d}$ (0.100g, $0.7 \mathrm{mmol})$, 4-amidinium-1,2-phenylenediamine $11 \mathrm{a}(0.135 \mathrm{~g}, 0.7 \mathrm{mmol})$ and $p$-benzoquinone $(0.078 \mathrm{~g}, 0.7 \mathrm{mmol})$ in absolute ethanol $(3.5 \mathrm{~mL})$ was obtained $0.023 \mathrm{~g}(10.4 \%)$ of violet solid; $\mathrm{mp}>300{ }^{\circ} \mathrm{C} ;{ }^{1} \mathrm{H}$ NMR (300 MHz, DMSO-d $\left.d_{6}\right) \delta / p p m: 13.46$ (bs, $1 \mathrm{H},-\mathrm{OH}$ ), 12.73 (bs, 1H, Bim-NH), 10.16 (bs, $1 \mathrm{H},-\mathrm{OH}$ ), 9.30 (bs, 2H, $\left.\mathrm{C}\left(\mathrm{NH}_{2}\right)_{2}{ }^{+}\right), 8.99$ (bs, $\left.2 \mathrm{H},-\mathrm{C}\left(\mathrm{NH}_{2}\right)_{2}{ }^{+}\right), 8.12(\mathrm{bs}, 1 \mathrm{H}, \mathrm{Ar}-\mathrm{H}), 7.99$ (d, $1 \mathrm{H}, J=8.5 \mathrm{~Hz}, \operatorname{Ar}-H), 7.77(\mathrm{~d}, 1 \mathrm{H}, J=7.7 \mathrm{~Hz}, \operatorname{Ar}-H), 7.68(\mathrm{~d}, 1 \mathrm{H}$, $J=8.6 \mathrm{~Hz}, \mathrm{Ar}-H), 6.49(\mathrm{dd}, 1 \mathrm{H}, J=8.5 \mathrm{~Hz}, J=2.2 \mathrm{~Hz}, \mathrm{Ar}-H), 6.45$ (d, $1 \mathrm{H}, J=2.1 \mathrm{~Hz}, \mathrm{Ar}-H) ;{ }^{13} \mathrm{C} \mathrm{NMR}\left(75 \mathrm{MHz}\right.$, DMSO- $\left.d_{6}\right) \delta / \mathrm{ppm}$ : 166.0, 165.9, 161.7, 161.5, 160.1, 159.9, 155.5, 154.8, 145.0, $140.8,137.2,133.0,128.6,128.4,122.3,122.0,121.4,121.3$, $117.8,117.5,111.7,111.6,108.0$ (2C), 104.1, 104.0, 103.0 (2C); LC-MS (ESI) $\mathrm{m} / \mathrm{z}: 269.1\left[\left(\mathrm{M}-\mathrm{Cl}^{-}\right)^{+}\right]$.

\section{5(6)-(4,5-Dihydro-1H-imidazol-3-ium-2-yl)-2-(4-} cyanophenyl)benzimidazole chloride 16

Using above described method from 4-cyanobenzaldehyde 1a (0.075 g, $0.6 \mathrm{mmol}), 2$-(3,4-diaminophenyl)-4,5-dihydro$1 \mathrm{H}$-imidazol-3-ium chloride $11 \mathrm{~b}(0.121 \mathrm{~g}, 0.6 \mathrm{mmol})$ and p-benzoquinone $(0.062 \mathrm{~g}, 0.6 \mathrm{mmol})$ in absolute ethanol $(4 \mathrm{~mL})$ was obtained $0.112 \mathrm{~g}$ (72\%) of grey solid; $\mathrm{mp} 235$ $237{ }^{\circ} \mathrm{C}$; ${ }^{1} \mathrm{H}$ NMR (300 MHz, DMSO- $d_{6}$ ) $\delta /$ ppm: 14.13 (bs, $1 \mathrm{H}, \mathrm{Bim}-\mathrm{NH}), 10.64\left(\mathrm{~s}, 2 \mathrm{H},-\mathrm{C}(\mathrm{NH}-)_{2}{ }^{+}\right), 8.43(\mathrm{~d}, 2 \mathrm{H}, J=8.4 \mathrm{~Hz}$, Ar-H), $8.19(\mathrm{~d}, 1 \mathrm{H}, J=8.4 \mathrm{~Hz}, \operatorname{Ar}-\mathrm{H}), 8.05(\mathrm{~d}, 2 \mathrm{H}, J=8.5 \mathrm{~Hz}$, Ar-H), $7.98(\mathrm{~d}, 1 \mathrm{H}, J=8.3 \mathrm{~Hz}, \mathrm{Ar}-\mathrm{H}), 7.86(\mathrm{~s}, 1 \mathrm{H}, \mathrm{Ar}-H), 4.01$ $\left(\mathrm{s}, 4 \mathrm{H},-\mathrm{CH}_{2} \mathrm{CH}_{2}-\right) ;{ }^{13} \mathrm{C}$ NMR (150 MHz, DMSO- $\left.d_{6}\right) \delta / \mathrm{ppm}$ : 165.3, 164.4, 150.3, 142.2, 134.1, 133.3, 133.0 (2C), 132.7, $129.4,127.6,118.4,115.6,113.2,112.8$. LC-MS (ESI) $\mathrm{m} / \mathrm{z}$ : $288.6\left[\left(\mathrm{M}-\mathrm{Cl}^{-}\right)^{+}\right]$.

\section{5(6)-(4,5-Dihydro-1H-imidazol-3-ium-2-yl)-2-(4- chlorophenyl)benzimidazole chloride 17}

Using above described method from 4-chlorobenzaldehyde 1b (0.075 g, $0.5 \mathrm{mmol}), 2$-(3,4-diaminophenyl)-4,5-dihydro$1 \mathrm{H}$-imidazol-3-ium chloride $11 \mathrm{~b}(0.113 \mathrm{~g}, 0.5 \mathrm{mmol})$ and p-benzoquinone $(0.057 \mathrm{~g}, 0.5 \mathrm{mmol})$ in absolute ethanol $(4 \mathrm{~mL})$ was obtained $0.124 \mathrm{~g}$ (70\%) of violet solid; $\mathrm{mp} 260$ $262{ }^{\circ} \mathrm{C} ;{ }^{1} \mathrm{H}$ NMR $\left(600 \mathrm{MHz}\right.$, DMSO- $\left.d_{6}\right) \delta / \mathrm{ppm}: 14.02(\mathrm{~s}, 1 \mathrm{H}$, Bim-NH), 10.68 (bs, 2H, $\left.-\mathrm{C}(\mathrm{NH}-)_{2}{ }^{+}\right), 8.41(\mathrm{~s}, 1 \mathrm{H}, \mathrm{Ar}-\mathrm{H}), 8.30$ (d, $2 \mathrm{H}, J=7.6 \mathrm{~Hz}, \mathrm{Ar}-H), 7.88$ (bs, $1 \mathrm{H}, \mathrm{Ar}-H), 7.81(\mathrm{~d}, 1 \mathrm{H}, J=7.8$ $\mathrm{Hz}, \mathrm{Ar}-\mathrm{H}), 7.70$ (d, $2 \mathrm{H}, \mathrm{J}=8.6 \mathrm{~Hz}, \mathrm{Ar}-\mathrm{H}), 4.03\left(\mathrm{~s}, 4 \mathrm{H},-\mathrm{CH}_{2} \mathrm{CH}_{2}\right)$; ${ }^{13} \mathrm{C}$ NMR (75 MHz, DMSO- $\left.d_{6}\right) \delta /$ ppm: 165.9, 165.7, 150.1, 135.9, 129.7, 129.6 (2C), 129.2 (2C), 128.6, 123.4, 116.1, 115.9, 45.0 (2C); LC-MS (ESI) m / z: 298.2 [(M-Cl- $\left.)^{+}\right]$.

5(6)-(4,5-Dihydro-1H-imidazol-3-ium-2-yl)-2-(4trifluoromethylphenyl)benzimidazole chloride 18 Using above described method from 4-trifluoromethylbenzaldehide 1c $(0.075 \mathrm{~g}, 0.4 \mathrm{mmol}), 2$-(3,4-diaminophenyl)-4,5-dihydro- $1 \mathrm{H}$-imidazol-3-ium chloride $\mathbf{1 1 b}$ (0.091 $\mathrm{g}, 0.4 \mathrm{mmol}$ ) and $p$-benzoquinone $(0.046 \mathrm{~g}, 0.4 \mathrm{mmol})$ in absolute ethanol $(4 \mathrm{~mL}$ ) was obtained $0.097 \mathrm{~g}(61 \%)$ of dark 
grey solid; mp 281-283 ${ }^{\circ} \mathrm{C} ;{ }^{1} \mathrm{H}$ NMR (600 MHz, DMSO- $d_{6}$ ) $\delta /$ ppm: 14.20 (bs, $1 \mathrm{H}, \mathrm{Bim}-\mathrm{NH}), 10.74$ (bs, $\left.2 \mathrm{H},-\mathrm{C}(\mathrm{NH}-)_{2}{ }^{+}\right)$, $8.51(\mathrm{~d}, 2 \mathrm{H}, J=8.4 \mathrm{~Hz}, \operatorname{Ar}-\mathrm{H}), 8.42(\mathrm{~s}, 1 \mathrm{H}, \mathrm{Ar}-\mathrm{H}), 7.97(\mathrm{~d}, 2 \mathrm{H}$, $J=8.5 \mathrm{~Hz}, \operatorname{Ar}-H), 7.94-7.82(\mathrm{~m}, 2 \mathrm{H}, \operatorname{Ar}-H), 4.02(\mathrm{~s}, 4 \mathrm{H},-$ $\mathrm{CH}_{2} \mathrm{CH}_{2}$ ); ${ }^{13} \mathrm{C}$ NMR $\left(75 \mathrm{MHz}\right.$, DMSO-d $\left.d_{6}\right) \delta / \mathrm{ppm}: 165.9$, $165.5,147.9,133.7,132.5,130.7$ (q), 128.4 (2C), 126.6 (2C), 125.8, 123.2, 116.1, 113.2, 44.9 (2C); LC-MS (ESI) $\mathrm{m} / \mathrm{z}$ : $331.6\left[(\mathrm{M}-\mathrm{Cl})^{+}\right]$.

\section{5(6)-(4,5-Dihydro-1H-imidazol-3-ium-2-yl)-2-(2,4-} dihydroxyphenyl)benzimidazole chloride 19[13] Using above described method from 2,4-dihydroxybenzaldehyde 1d $(0.080 \mathrm{~g}, 0.6 \mathrm{mmol}), 2$-(3,4-diaminophenyl)-4,5-dihydro- $1 \mathrm{H}$-imidazol-3-ium chloride $11 \mathbf{b}$ ( 0.123 $\mathrm{g}, 0.6 \mathrm{mmol}$ ) and $p$-benzoquinone $(0.063 \mathrm{~g}, 0.6 \mathrm{mmol})$ in absolute ethanol $(3.5 \mathrm{~mL}$ ) was obtained $0.035 \mathrm{~g}(18.2 \%)$ of grey-violet solid; $\mathrm{mp}>300{ }^{\circ} \mathrm{C} ;{ }^{1} \mathrm{H}$ NMR $\left(600 \mathrm{MHz}\right.$, DMSO- $\left.d_{6}\right)$ $\delta /$ ppm: 13.70 (bs, $1 \mathrm{H},-\mathrm{OH}$ ), 13.58 (bs, $1 \mathrm{H},-\mathrm{OH}), 12.75$ (s, 1H, Bim-NH), 12.58 (s, 1H, Bim-NH), 10.56 (bs, 4H, -C(NH$\left.\mathrm{l}^{+}{ }^{+}\right), 10.23(\mathrm{~s}, 1 \mathrm{H},-\mathrm{OH}), 10.21(\mathrm{~s}, 1 \mathrm{H},-\mathrm{OH}), 8.35(\mathrm{~s}, 1 \mathrm{H}, \mathrm{Ar}-\mathrm{H})$, $8.24(\mathrm{~s}, 1 \mathrm{H}, \mathrm{Ar}-H), 8.06(\mathrm{~d}, 1 \mathrm{H}, J=8.3 \mathrm{~Hz}, \operatorname{Ar}-H), 7.99(\mathrm{~d}, 1 \mathrm{H}, J$ $=7.1 \mathrm{~Hz}, \mathrm{Ar}-H), 7.85-7.82(\mathrm{~m}, 3 \mathrm{H}, \mathrm{Ar}-H), 7.77(\mathrm{~d}, 1 \mathrm{H}, J=7.2$ $\mathrm{Hz}, \mathrm{Ar}-\mathrm{H}), 6.49$ (d, $2 \mathrm{H}, \mathrm{J}=8.6 \mathrm{~Hz}, \mathrm{Ar}-\mathrm{H}), 6.45(\mathrm{~s}, 2 \mathrm{H}, \mathrm{Ar}-\mathrm{H}), 4.02$ (s, $8 \mathrm{H},-\mathrm{CH}_{2} \mathrm{CH}_{2}$ ) $;{ }^{13} \mathrm{C}$ NMR (75 MHz, DMSO-d $\left.d_{6}\right) \delta / \mathrm{ppm}$ : 165.8, 162.2, 160.4, 129.1, 123.1, 115.9, 108.5 (2C), 104.5, 103.5 (2C), 44.7 (2C); LC-MS (ESI) $\mathrm{m} / \mathrm{z}: 298.2$ [(M-Cl-) $)^{+}$.

\section{Antiproliferative Activity}

The experiments were carried out on three human cell lines: HCT 116 (colon carcinoma), H 460 (lung carcinoma) and MCF-7 (breast carcinoma) according to the previously published experimental procedure. ${ }^{[15,17]}$

Briefly, the cells were grown in DMEM medium with the addition of $10 \%$ fetal bovine serum (FBS), $2 \mathrm{mM} \mathrm{L-}$ glutamine, $100 \mathrm{U} \mathrm{mL}$ penicillin and $100 \mu \mathrm{g} \mathrm{mL}-1$ streptomycin, and cultured as monolayers at $37{ }^{\circ} \mathrm{C}$ in a humidified atmosphere with $5 \% \mathrm{CO}_{2}$. Cells were seeded at $2 \times 10^{3}$ cells/well in a standard 96-well microtiter plates and left to attach for $24 \mathrm{~h}$. Next day, test compound was added in five serial 10 -fold dilutions. The cell growth rate was evaluated after $72 \mathrm{~h}$ of incubation, using MTT assay. Obtained results are expressed as $I \mathrm{I}_{50}$ value which stands for the concentration of the compound necessary for $50 \%$ of growth inhibition. The $\mathrm{IC}_{50}$ values are calculated from concentration-response curve using linear regression analysis by fitting the test concentrations that give PG values above and below the reference value (i.e. $50 \%$ ). Each test was performed in quadruplicate in at least two individual experiments.

\section{RESULTS AND DISCUSSION}

\section{Chemistry}

The targeted amidino substituted benzothiazoles 3-10 were synthesized according to the procedure shown in Scheme 1 by using conventional methods for construction of structurally related fused heteroaromatic derivatives. Starting from commercially available substituted benzaldehydes 1a-1d and amidino substituted benzenethiolates $\mathbf{2 a - 2} \mathbf{b}$, cyclocondensation in refluxing acetic acid followed by quenching with hydrochloric acid, afforded benzothiazoles $\mathbf{3 - 1 0}$ as hydrochloride salts in moderate to good reaction yields. This method has been found to be efficient for direct condensation of thermally and acid stable aldehydes whith amidino substituted 2-aminothiophenoles without using any catalyst or oxidant. The precursors $\mathbf{2 a -}$ $\mathbf{2} \mathbf{b}$ as zwitterions were prepared by Pinner reaction from 6cyanobenzothiazole according to our previously described and optimized method. ${ }^{[27]}$

Amidino substituted benzimidazole derivatives 1219 were prepared following the experimental protocol as shown in the Scheme 2. Within the reaction of cyclocondensation, from substituted benzaldehydes $\mathbf{1 a - 1 d}$ and 4amidino/4-(2-imidazolinyl)-1,2-phenylene-diamines hydrochlorides 11a-11b, corresponding 2-phenyl substituted benzimidazoles $\mathbf{1 2 - 1 9}$ as hydrochloride salts were prepared in moderate reaction yields. Amidines 11a-11b

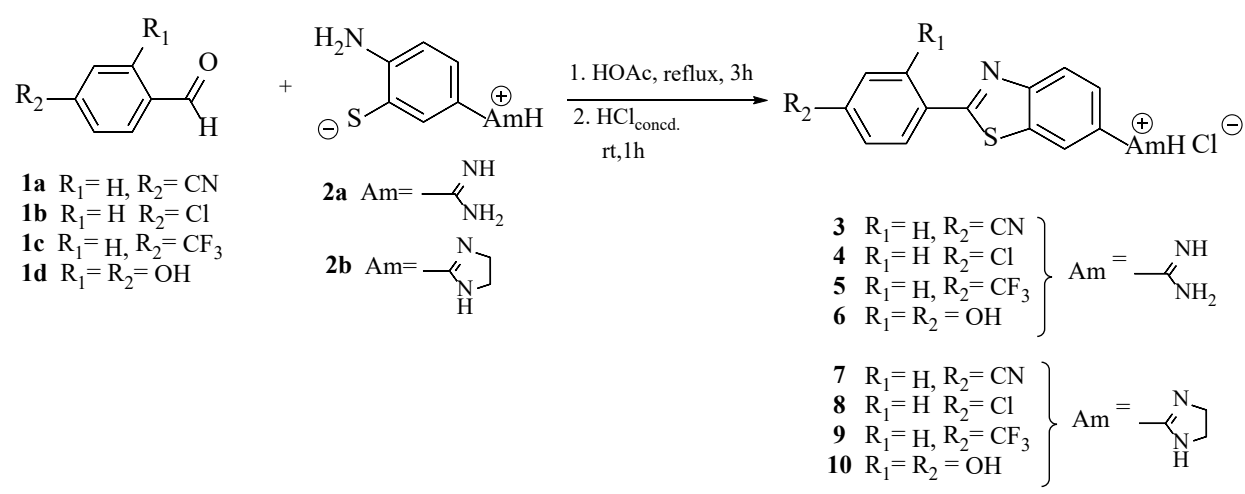

Scheme 1. Synthesis of amidino substituted benzothiazole derivatives 3-10. 


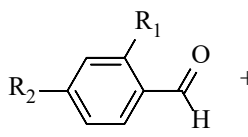

1a $\mathrm{R}_{1}=\mathrm{H}, \mathrm{R}_{2}=\mathrm{CN}$ 1b $\mathrm{R}_{1}=\mathrm{H} \mathrm{R} \mathrm{R}_{2}=\mathrm{Cl}$

1c $\mathrm{R}_{1}=\mathrm{H}, \mathrm{R}_{2}=\mathrm{CF}_{3}$ 1d $\mathrm{R}_{1}=\mathrm{R}_{2}=\mathrm{OH}$

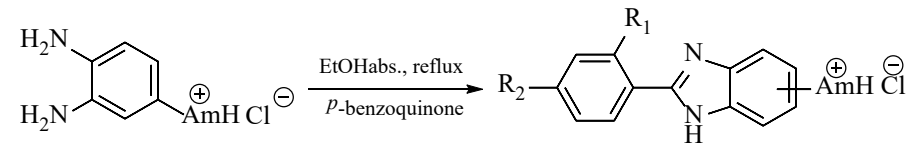

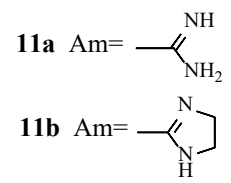

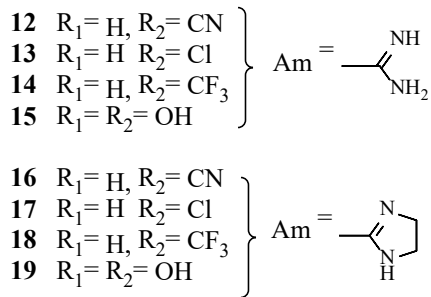

Scheme 2. Synthesis of amidino substituted benzimidazole derivatives 12-19.

obtained in the acidic Pinner reaction from corresponding cyano substituted precursors according to the previously published procedures. ${ }^{[17]}$

The structures of all newly prepared benzothiazole and benzimidazole derivatives were determined by using ${ }^{1} \mathrm{H}$ and ${ }^{13} \mathrm{C}$ NMR spectroscopy and mass spectrometry. NMR analysis based on the values of $\mathrm{H}-\mathrm{H}$ coupling constants and chemical shifts in the ${ }^{1} \mathrm{H}$ spectra confirmed the structures of compounds. Furthermore, ${ }^{13} \mathrm{C}$ NMR chemical shifts were consistent with the suggested structures.

The appearance of protons related to unsubstituted and cyclic amidino substituent in the aliphatic part can be observed in ${ }^{1} \mathrm{H}$ for unsubstituted and both in ${ }^{1} \mathrm{H}$ and ${ }^{13} \mathrm{C}$ NMR for 2-imidazolinyl amidine. IR spectroscopy was used for the monitoring of Pinner reaction due to the synthesis of main precursors $\mathbf{2 a}, \mathbf{2} \mathbf{b}, \mathbf{1 1} \mathbf{a}$ and $\mathbf{1 1} \mathbf{b}$.

\section{Antiproliferative Activity}

In order to assess their antiproliferative activity in vitro, all prepared benzothiazole and benzimidazole derivatives were tested against HCT116 (colon carcinoma), H460 (lung carcinoma) and MCF-7 (breast carcinoma) cancer cell lines. For comparison reasons, a reference compound, etoposide was used (Table 1).

The synthesized compounds were designed in order to study the influence of the heteroatom in benzazole nuclei, the type of substituent placed on the phenyl ring and the type of the amidino group attached on the benzazole nuclei on antiproliferative activity in vitro and selectivity towards three cancer cells. Obtained results revealed that the type of benzazole nuclei significantly influenced the antiproliferative effect on tested cells, thus confirming the influence of the heteroatom on the antiproiliferative activity (Fig. 3). In general, most of the tested compounds showed moderate antiproliferative effect, while benzothiazole derivatives 3-10 displayed significantly better activity in comparison to their benzimidazole analogues 12-19. Among all tested benzothiazole amidines, there was no notable difference in the antiproliferative activity between amidino substituted 3-6 and 2-imidazolinyl substituted 7-10 derivatives with the exception of 2,4-dihydroxy substituted derivatives 6 and 10. Amidino substituted benzothiazole 6 did not show any activity while its 2-imidazolinyl analogue $\mathbf{1 0}$ showed moderate antiproliferative effect with selective activity toward H 460 cancer cell. On the other hand, all tested benzimidazole derivatives showed lower antiproliferative activity in comparison to their benzothiazole analogues.

Unlike benzothiazole amidines 3-10, the antiproliferative activity of benzimidazole amidines 12-19, in general was influenced by the type of attached amidino group. 2-Imidazolinyl substituted derivatives 16-18 showed a slightly improvement of antiproliferative effect in comparison to their amidino substituted analogues 12-14 with some selectivity against MCF-7 cells, especially for derivatives $\mathbf{1 7}$ and $\mathbf{1 8}$.

Surprisingly, the activity of derivatives $\mathbf{1 5}$ and 19, substituted with two hydroxy groups was not influenced by the type of the amidino group.

In conclusion, taking into account the obtained results, it could be concluded that benzazole scaffold, especially benzothiazole nuclei, offer a great potential for further optimization of the structure which could lead to more active and selective benzothiazole derivatives as a promising antiproliferative agents.

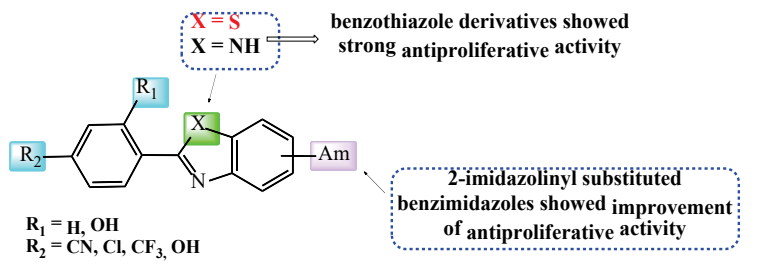

Figure 3. Insights into SAR for antiproliferative activity in vitro of benzazole derivatives. 
Table 1. $I C_{50}{ }^{(a)}$ values of 2-phenylbenzothiazoles 3-10 and 2-phenylbezimidazoles 12-19.<smiles></smiles>

\begin{tabular}{|c|c|c|c|c|c|c|c|}
\hline \multirow{2}{*}{ Cpd } & \multirow{2}{*}{$\mathrm{R}_{1}$} & \multirow{2}{*}{$\mathrm{R}_{2}$} & \multirow{2}{*}{$x$} & \multirow{2}{*}{$\mathrm{Am}$} & \multicolumn{3}{|c|}{ Cell lines } \\
\hline & & & & & НСТ116 & MCF-7 & H 460 \\
\hline 3 & $\mathrm{H}$ & $\mathrm{CN}$ & S & unsubstituted & $2.4 \pm 0.08$ & $1.9 \pm 0.4$ & $1.9 \pm 0.03$ \\
\hline 4 & $\mathrm{H}$ & $\mathrm{Cl}$ & S & unsubstituted & $2.5 \pm 0.8$ & $1.7 \pm 0.26$ & $1.5 \pm 0.2$ \\
\hline 5 & $\mathrm{H}$ & $\mathrm{CF}_{3}$ & $S$ & unsubstituted & $2.7 \pm 1.9$ & $1.7 \pm 0.3$ & $1.5 \pm 0.3$ \\
\hline 6 & $\mathrm{OH}$ & $\mathrm{OH}$ & $S$ & unsubstituted & $>100$ & $>100$ & $>100$ \\
\hline 7 & $\mathrm{H}$ & $\mathrm{CN}$ & S & 2-imidazolinyl & $2.6 \pm 0.6$ & $1.7 \pm 0.4$ & $2.8 \pm 0.9$ \\
\hline 8 & $\mathrm{H}$ & $\mathrm{Cl}$ & S & 2-imidazolinyl & $2.5 \pm 1.2$ & $1.6 \pm 0.4$ & $1.25 \pm 0.07$ \\
\hline 9 & $\mathrm{H}$ & CF3 & S & 2-imidazolinyl & $1.6 \pm 0.5$ & $1.6 \pm 0.5$ & $1.5 \pm 0.2$ \\
\hline 10 & $\mathrm{OH}$ & $\mathrm{OH}$ & S & 2-imidazolinyl & $27 \pm 12$ & $21 \pm 4$ & $5 \pm 1.4$ \\
\hline 12 & $\mathrm{H}$ & $\mathrm{CN}$ & $\mathrm{NH}$ & unsubstituted & $>100$ & $55 \pm 12$ & $>100$ \\
\hline 13 & $\mathrm{H}$ & $\mathrm{Cl}$ & $\mathrm{NH}$ & unsubstituted & $67 \pm 2.5$ & $12 \pm 0.4$ & $81 \pm 4$ \\
\hline 14 & $\mathrm{H}$ & $\mathrm{CF}_{3}$ & $\mathrm{NH}$ & unsubstituted & $65 \pm 5$ & $16 \pm 1.3$ & $>100$ \\
\hline 15 & $\mathrm{OH}$ & $\mathrm{OH}$ & $\mathrm{NH}$ & unsubstituted & $>100$ & $44 \pm 0.5$ & $>100$ \\
\hline 16 & $\mathrm{H}$ & $\mathrm{CN}$ & $\mathrm{NH}$ & 2-imidazolinyl & $65 \pm 5$ & $16 \pm 1.3$ & $>100$ \\
\hline 17 & $\mathrm{H}$ & $\mathrm{Cl}$ & $\mathrm{NH}$ & 2-imidazolinyl & $7.5 \pm 2.5$ & $5 \pm 1.7$ & $14 \pm 1.6$ \\
\hline 18 & $\mathrm{H}$ & $\mathrm{CF}_{3}$ & $\mathrm{NH}$ & 2-imidazolinyl & $9 \pm 0.9$ & $6 \pm 2$ & $15 \pm 3$ \\
\hline 19 & $\mathrm{OH}$ & $\mathrm{OH}$ & $\mathrm{NH}$ & 2-imidazolinyl & $>100$ & $42 \pm 1.2$ & $>100$ \\
\hline Etoposide & & $5 \pm 2$ & $1 \pm 0.7$ & $0.1 \pm 0.04$ & & & \\
\hline
\end{tabular}

(a) The concentration that causes $50 \%$ growth inhibition.

\section{CONCLUSION}

As a continuation of our scientific research based on the biologically active amidino substituted benazazoles, herein we present the design and synthesis of 2-phenyl substituted benzothiazole and benzimidazole derivatives bearing two types of amidino groups, either unsubstituted or cyclic amidine.

The mentioned compounds were synthesized to explore their antiproliferative activity in vitro on several cancer cells while results were compared to standard antiproliferative agent etoposide.

Additionally, our mail goal was also to study SAR and the influence of the type of substituent placed at the phenyl ring, the type of the benzazole nuclei as well as the type of the amidino group placed at the heteroaromatic nuclei on the antiproliferative activity.

The obtained results revealed that the majority of tested compounds showed moderate to high antiproliferative activity without significant selectivity between tested cell lines. The highest impact on the antiproliferative activity was shown by the type of the benzazole nuclei which led to the fact that all benzothiazole derivatives 3-5 and 7-10, with the exception of dihydroxy substituted derivative $\mathbf{6}$, showed improvement of antiproliferative activity in comparison to their benzimidazole analogues.

Additionally, the type of the attached amidino group on benzazole nuclei and substituents on the phehyl ring did not have the significant impact on the antiproliferative activity of tested benzothiazole derivatives with the exception of dihydroxy substituted derivatives $\mathbf{6}$ and $\mathbf{1 0}$. Oppositely, among the benzimidazole derivatives, 2imidazolinyl substituted derivatives $\mathbf{1 6 - 1 8}$ showed slightly enhancement of antiproliferative effect in comparison to amidino substituted analogues 12-14 with the exception of dihydroxy derivatives $\mathbf{1 5}$ and $\mathbf{1 9}$.

All obtained results revealed that this type of synthesized benzazoles, especially benzothiazole derivatives, has a high potential for further optimization and improvement of their antiproliferative activity and selectivity against chosen cancer cell. 
Acknowledgment. We greatly appreciate the financial support of the Croatian Science Foundation under the projects 4379 entitled Exploring the antioxidative potential of benzazole scaffold in the design of novel antitumor agents. The authors have declared no conflict of interest.

List od Abbreviations.

TMS - tetramethylsilane

DMSO - dimethylsulfoxide

DMEM - Dulbecco's Modified Eagle Medium

MTT - 3-[4,5-dimethylthiazole-2-yl]-2,5-diphenyltetrazolium bromide

Supplementary Information. Supporting information to the paper is attached to the electronic version of the article at: https://doi.org/10.5562/cca3531.

PDF files with attached documents are best viewed with Adobe Acrobat Reader which is free and can be downloaded from Adobe's web site.

\section{REFERENCES}

[1] R. B. Silverman, The Organic Chemistry of Drug Design and Drug Action, Elsevier Academic Press, Amsterdam, 2004.

[2] W. D. Wilson, B. Nguyen, F. A. Tanious, A. Mathis, J. E. Hall, C. E. Stephens, D. W. Boykin, Curr. Med. Chem. Anticancer Agents, 2005, 5, 389-408. https://doi.org/10.2174/1568011054222319

[3] Y. Bansal, O. Silakari, Bioorg. Med. Chem., 2012, 20, 6208-6236.

https://doi.org/10.1016/j.bmc.2012.09.013

[4] P. C. Sharma, A. Sinhmar, A. Sharma, H. Rajak, D. Pal Pathak, J. Enzyme Inhib. Med. Chem., 2013, 28, 240266. https://doi.org/10.3109/14756366.2012.720572

[5] B. Zhou, B. Li, W. Yi, X. Bu, L. Ma, Bioorg. Med. Chem. Lett., 2013, 23, 3759-3763.

https://doi.org/10.1016/j.bmcl.2013.05.004

[6] S. Saeed, N. Rasshid, P. Jones, M. Ali, R. Hussain, Eur. J. Med. Chem., 2010, 45, 1323-1331.

https://doi.org/10.1016/j.ejmech.2009.12.016

[7] L. Racané, M. Sedić, N. Ilić, M. Aleksić, S. Kraljević Pavelić, G. Karminski-Zamola, Anti-Cancer Agents Med. Chem. 2017, 17, 57-66.

[8] K. Shah, S. Chhabra, S. K. Shrivastava, P. Mishra. Med. Chem. Res., 2013, 22, 5077-5104. https://doi.org/10.1007/s00044-013-0476-9

[9] K. Starčević, M. Kralj, K. Ester, I. Sabol, M. Grce, K. Pavelić, G. Karminski-Zamola, Bioorg. Med. Chem., 2007, 15, 4419-4426. https://doi.org/10.1016/j.bmc.2007.04.032

[10] C. Papadopoulou, A. Geronikaki, D. HadjipavlouLitina, I/ Farmaco 2005, 60, 969-973. https://doi.org/10.1016/j.farmac.2005.06.014
[11] J. Velík, V. Baliharová, J. Fink-Gremmels, S. Bull, J. Lamka, L. Skálová, Res. Vet. Sci., 2004, 76, 95-108. https://doi.org/10.1016/j.rvsc.2003.08.005

[12] R. Likhar, P. Perumal, N. Kolhe, V. H. Bhaskar, P. Daroi, Int. J. Curr. Pharm. Res., 2015, 7, 34-37.

[13] L. Racané, M. Cindrić, N. Perin, P. Roškarić, K. Starčević, T. Mašek, M. Maurić, J. Dogan, G. Karminski-Zamola, Croat. Chem. Acta, 2017, 90, 187-195. https://doi.org/10.5562/cca3146

[14] A. Rescifina, C. Zagni, M.G. Varrica, V. Pistarà, A. Corsaro, Eur. J. Med. Chem., 2014, 74, 95-115. https://doi.org/10.1016/j.ejmech.2013.11.029

[15] N. Perin, I. Martin-Kleiner, R. Nhili, W. Laine, M. H. David-Cordonnier, O. Vugrek, G. Karminski-Zamola, M. Kralj, M. Hranjec, Med. Chem. Commun., 2013, 4, 1537-1550. https://doi.org/10.1039/c3md00193h

[16] L. Racané, V. Tralić-Kulenović, S. Kraljević Pavelić, I. Ratkaj, P. Peixoto, R. Nhili, S. Depauw, M. P. Hildebrand, M.-H. David-Cordonnier, K. Pavelić, G. Karminski-Zamola, J. Med. Chem., 2010, 53, 24182432. https://doi.org/10.1021/jm901441b

[17] M. Hranjec, M. Kralj, I. Piantanida, M. Sedić, L. Šuman, K. Pavelić, G. Karminski-Zamola, J. Med. Chem., 2007, 50, 5696-5711.

https://doi.org/10.1021/jm070876h

[18] L. Racané, M. Kralj, L. Šuman, R. Stojkovic, V. TralicKulenovic, G. Karminski-Zamola, Bioorg. Med. Chem., 2010, 18, 1038-1044.

https://doi.org/10.1016/j.bmc.2009.12.054

[19] W. D. Wilson, B. Nguyen, F. Tanious, A. Mathis, J. E. Hall, C. Stephens, D. W. Boykin, Curr. Med. Chem. Anticancer Agents, 2005, 5, 389-408.

https://doi.org/10.2174/1568011054222319

[20] M. Demeunynck, C. Bailly, W. D. Wilson, In DNA and RNA Binders, Wiley-VCH, Weinheim, 2002.

[21] M. Demeunynck, C. Bailly, W. D. Wilson, Small Molecule DNA and RNA Binders: From Synthesis to Nucleic Acid Complexes, Wiley-VCH, Weinheim, 2003. https://doi.org/10.1002/3527601783

[22] A. Patel, H. J. Smith, J. Sturzebecher, in Introduction to the Principles of Drug Design and Action (Ed.: H. J. Smith) Harwood Academic Publishers, 2005.

[23] F. A. Tanious, D. Hamelberg, C. Bailly, A. Czarny, D. W. Boykin, W. D. Wilson, J. Am. Chem. Soc., 2004, 126, 143-153. https://doi.org/10.1021/ja030403+

[24] M. Hranjec, I. Piantanida, M. Kralj, L. Šuman, K. Pavelić, G. Karminski-Zamola, J. Med. Chem., 2008, 51, 4899-4910. https://doi.org/10.1021/jm8000423

[25] L. Racané, R. Stojković, V. Tralić- Kulenović, H. Cerić, M. Đaković, K. Ester, A. Mišir Krpan, M. Radić Stojković, Eur. J. Med. Chem., 2014, 86, 406-419. https://doi.org/10.1016/j.ejmech.2014.08.072 
[26] M. Cindrić, S. Jambon, A. Harej, S. Depauw, M. DavidCordonnier, S. Kraljević Pavelić, G. Karminski-Zamola, M. Hranjec, Eur. J. Med. Chem., 2017, 136, 468-479. https://doi.org/10.1016/j.ejmech.2017.05.014

[27] L. Racané, V. Tralić-Kulenović, Z. Mihalić, G. Pavlović, G. Karminski-Zamola, Tetrahedron, 2008,
64, 11594-11602.

https://doi.org/10.1016/j.tet.2008.10.026

[28] T. A. Fairley, R. R. Tidwell, I. Donkor, N. A. Naiman, K. A. Ohemeng, R. J. Lombardy, J. A. Bentley, M. Cory, J. Med. Chem., 1993, 36, 1746-1753.

https://doi.org/10.1021/jm00064a008 